\title{
On the Solar Cycle Variation of the Solar Diurnal Anisotropy of Multi-TeV Cosmic-ray Intensity Observed with the Tibet Air Shower Array
}

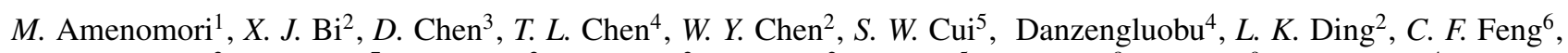

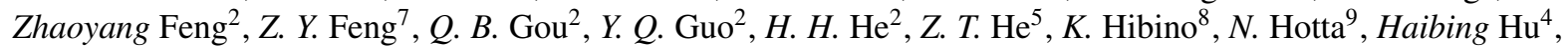
H. B. $\mathrm{Hu}^{2}, J$. Huang ${ }^{2}, H . Y . \mathrm{Jia}^{7}, L . \mathrm{Jiang}^{2}, F . \mathrm{Kajino}^{10}, K . \mathrm{Kasahara}^{11}, Y$ Katayose $^{12}, C . \mathrm{Kato}^{13}, K . \mathrm{Kawata}^{14}, M . \mathrm{Kozai}^{13,15}$, Labaciren ${ }^{4}$, G. M. Le ${ }^{16}, A . F . \mathrm{Li}^{17,6,2}, H . J . \mathrm{Li}^{4}, W . J . \mathrm{Li}^{2,7}, Y . H . \mathrm{Lin}^{2,18, *}, C . \mathrm{Liu}^{2}, J . S . \mathrm{Liu}^{2}, M . Y . \mathrm{Liu}^{4}, H . \mathrm{Lu}^{2}$, $X . R$. Meng $^{4}, T$. Miyazaki $^{13}, K$. Munakata $^{13}, T$. Nakajima $^{13}, Y$. Nakamura $^{13}, H$. Nanjo $^{1}, M$. Nishizawa $^{19}, T$. Niwa $^{13}$,

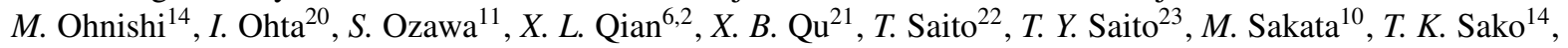

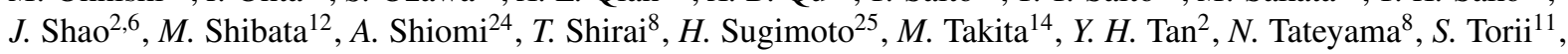
H. Tsuchiya ${ }^{26}, S . \mathrm{Udo}^{8}, H . \mathrm{Wang}^{2}, H . R$. Wu ${ }^{2}, L . \mathrm{Xue}^{6}, Y$. Yamamoto ${ }^{10}, K$. Yamauchi ${ }^{12}, Z$. Yang ${ }^{2}, A . F$. Yuan $^{4}, L . M . \mathrm{Zhai}^{3}$,

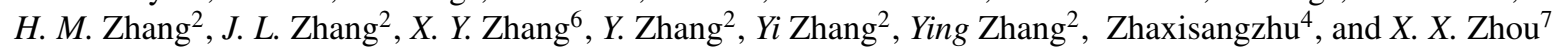
(The Tibet AS $\gamma$ Collaboration)

${ }^{1}$ Department of Physics, Hirosaki University, Hirosaki 036-8561, Japan

${ }^{2}$ Key Laboratory of Particle Astrophysics, Institute of High Energy Physics, Chinese Academy of Sciences, Beijing 100049, China

${ }^{3}$ National Astronomical Observatories, Chinese Academy of Sciences, Beijing 100012, China

${ }^{4}$ Department of Mathematics and Physics, Tibet University, Lhasa 850000, China

${ }^{5}$ Department of Physics, Hebei Normal University, Shijiazhuang 050016, China

${ }^{6}$ Department of Physics, Shandong University, Jinan 250100, China

${ }^{7}$ Institute of Modern Physics, SouthWest Jiaotong University, Chengdu 610031, China

${ }^{8}$ Faculty of Engineering, Kanagawa University, Yokohama 221-8686, Japan

${ }^{9}$ Faculty of Education, Utsunomiya University, Utsunomiya 321-8505, Japan

${ }^{10}$ Department of Physics, Konan University, Kobe 658-8501, Japan

${ }^{11}$ Research Institute for Science and Engineering, Waseda University, Tokyo 169-8555, Japan

${ }^{12}$ Faculty of Engineering, Yokohama National University, Yokohama 240-8501, Japan

${ }^{13}$ Department of Physics, Shinshu University, Matsumoto 390-8621, Japan

${ }^{14}$ Institute for Cosmic Ray Research, University of Tokyo, Kashiwa 277-8582, Japan

${ }^{15}$ Institute of Space and Astronautical Science, Japan Aerospace Exploration Agency (ISAS/JAXA), Sagamihara 252-5210, Japan

${ }^{16}$ National Center for Space Weather, China Meteorological Administration, Beijing 100081, China

${ }^{17}$ School of Information Science and Engineering, Shandong Agriculture University, Taian 271018, China

${ }^{18}$ University of Chinese Academy of Sciences, Beijing 100049, China

${ }^{19}$ National Institute of Informatics, Tokyo 101-8430, Japan

${ }^{20}$ Sakushin Gakuin University, Utsunomiya 321-3295, Japan

${ }^{21}$ College of Science, China University of Petroleum, Qingdao 266555, China

${ }^{22}$ Tokyo Metropolitan College of Industrial Technology, Tokyo 116-8523, Japan

${ }^{23}$ Max-Planck-Institut für Physik, Munich D-80805, Germany

${ }^{24}$ College of Industrial Technology, Nihon University, Narashino 275-8576, Japan

${ }^{25}$ Shonan Institute of Technology, Fujisawa 251-8511, Japan

${ }^{26}$ Japan Atomic Energy Agency, Tokai-mura 319-1195, Japan

\begin{abstract}
We analyze the temporal variation of the solar diurnal anisotropy of the multi-TeV cosmic-ray intensity observed with the Tibet air shower array from 2000 to 2009, covering the maximum and minimum of the 23rd solar cycle. We comfirm that a remarkable additional anisotropy component is superposed on the Compton-Getting anisotropy at $4.0 \mathrm{TeV}$, while its amplitude decreases at higher energy regions. In constrast to the additional anisotropy reported by the Matsushiro experiment at $0.6 \mathrm{TeV}$, we find the residual component measured by Tibet at multi-TeV energies is consistent with being stable, with a fairly constant amplitude of $0.041 \% \pm 0.003 \%$ and a phase at around 07:17 $\pm 00: 16$ local solar time at $4.0 \mathrm{TeV}$. This suggests the additional anisotropy observed by the Tibet experiment could result from mechanisms unrelated to solar activities.
\end{abstract}

\footnotetext{
*e-mail: linyh@ihep.ac.cn ORCID: 0000-0002-1767-6280
} 


\section{Introduction}

Back in 1935, Compton and Getting [1] proposed an apparent effect that the observer would see an enhanced intensity in the direction of his motion when he moves with respect to the isotropic cosmic-ray plasma in the rest frame, and a decreased intensity in the direction opposite to his motion. Later in 1968, Gleeson and Axford [2] applied this original idea to the terrestrial orbital motion around the Sun. If the cosmic ray (CR) energy spectrum is described by an inverse power law, this so-called Compton-Getting (CG) anisotropy in the solar time frame can be given by

$$
\frac{\Delta I}{I}=(\gamma+2) \frac{v}{c} \cos \theta
$$

where $\Delta I$ denotes the fractional $C R$ intensity enhancement due to the CG effect, $I$ the CR intensity, $\gamma$ the absolute value of the power-law index of the CR energy spectrum, $v=2.978 \times 10^{4} \mathrm{~m} \mathrm{~s}^{-1}$ the average orbital velocity of the Earth, $c$ the speed of light, and $\theta$ the angle between the direction of the incident cosmic rays and the direction of the detector's motion. Taking the latitude at the Yangbajing site into account, the amplitude of CG anisotropy should be decreased by a geometrical correction factor $F=0.829$ [3].

Over the last three decades, such a dipole solar anisotropy has been widely reported from sub-TeV to multi$\mathrm{TeV}$ energies (e.g., see [3-9]) . Both the amplitude and the phase expected from the CG anisotropy are consistent with the experimental observations above $10 \mathrm{TeV}$. Some results, nevertheless, indicate there exists a deviation from the theoretical prediction at lower energy regions. Amenomori et al. [5] reported a solar diurnal variation of $4.0 \mathrm{TeV}$ statistically deviates from the expected CG anisotropy at $8.3 \sigma$ in amplitude and at $5.3 \sigma$ in phase. Meanwhile, Munakata et al. [8] claimed an extra anisotropy superposed on the $\mathrm{CG}$ anisotropy at $0.6 \mathrm{TeV}$, with the constant phase at 15:00 local solar time (LT) and the amplitude varying from $0.008 \%$ to $0.043 \%$ which reveals a clear correlation with the solar activity. If these residual effects, indeed, both result from the solar modulation, a temporal variation of this additional anisotropy might also be observed by the Tibet experiment. In this paper, we present the analysis of the solar diurnal anisotropy for the multi-TeV cosmic-ray intensity with data collected by the Tibet air shower array from 2000 to 2009, covering the maximum and minimum of the 23 rd solar cycle, to examine the hypothesis mentioned above.

\section{Experiment and Data Analysis}

The Tibet air shower experiment is located at Yangbajing in Tibet, China (90.522 E, $30.102 \mathrm{~N} ; 4300 \mathrm{~m}$ above sea level; $606 \mathrm{~g} / \mathrm{cm}^{2}$ ). Originally constructed in 1990, the Tibet I array was a $7 \times 7$ matrix of 49 scintillation detectors deployed at a $15 \mathrm{~m}$ grid spacing [10]. This surface array has been gradually upgraded, by deploying additional counters to the preceding Tibet I, II and III arrays [11]. The current array, covering an effective area of $50,000 \mathrm{~m}^{2}$, consists of 761 fast-timing (FT) detectors, each $0.5 \mathrm{~m}^{2}$ in area, placed on a lattice of $7.5 \mathrm{~m}$ spacing, surrounded by 28 density (D) detectors. A lead plate of $0.5 \mathrm{~cm}$ thickness is put on the top of each counter to improve the fast-timing data by converting gamma rays in air showers into electron-positron pairs.

An event trigger signal is issued when any fourfold coincidence takes place in the FT counters recording more than 0.6 particles, resulting in the trigger rate $680 \mathrm{~Hz}$ at the threshold energy of a few $\mathrm{TeV}$. The primary cosmic-ray energy is estimated by $\sum \rho_{F T}$, which is defined as the sum of the number of particles per $\mathrm{m}^{2}$ for each FT detector. In this work, the data used for analysis were collected by the Tibet III array during 2095 live days from January 2000 to December 2009. CR events are selected after some simple criteria as follows: (1) software trigger condition of any fourfold coincidence in the FT counters recording more than 0.8 particles in charge, (2) zenith angle of the arrival direction less than $45^{\circ}$, (3) air shower core position located in the array, (4) $10 \leq \sum \rho_{F T}<27,27 \leq \sum \rho_{F T}<47$, $47 \leq \sum \rho_{F T}<178$, approximately corresponding to 4.0 $\mathrm{TeV}, 6.2 \mathrm{TeV}$ and $12.0 \mathrm{TeV}$ representative primary energy, respectively. In total, $2.5 \times 10^{10}$ events remain for further analysis.

To eliminate the spurious variation due to the atmospheric effects and possible detector biases, we adopt the following "East-West" method [12]. First of all, we classify the shower events in each hour at the solar time frame into "east" and "west" groups according to the geographical longitude of each event's incident direction. The daily variations of such two groups are defined as $E(t)$ and $W(t)$, respectively. On the other hand, we correct the number of events month by month to suppress the spurious anisotropy caused by the inconsistency of the observation, taking monthly variations of the observation live time into account. By taking the difference between $E(t)$ and $W(t)$, we can deduce the "differential" of the physical variation $R(t)$ as

$$
D(t) \equiv \frac{E(t)-W(t)}{2 \delta t}=\frac{R(t+\delta t)-R(t-\delta t)}{2 \delta t}=\frac{d}{d t} R(t),
$$

where $2 \delta t$ is the hour angle separation between the mean directions of east- and west-incident events. Comparing $D(t)$ with equation (1), we make a best fit of $D(t)$ with the harmonic function

$$
D^{f i t}(t)=A^{D} \cos \left[\frac{\pi}{12}\left(t-\Phi^{D}\right)\right]
$$

where $A^{D}$ and $\Phi^{D}$ are the amplitude and phase of the harmonic vector of $D(t)$. By "integrating" $D(t)$ in time $t$, we finally reach the corresponding amplitude and phase of $R(t)$, respectively, as

$$
\begin{aligned}
& A^{R}=\frac{12}{\pi} A^{D}, \\
& \Phi^{R}=\Phi^{D}+6 .
\end{aligned}
$$

Note that the errors of $A^{R}$ and $\Phi^{R}$ are deduced from errors of the observed $D(t)$.

\section{Results and Discussion}

To investigate the time variation of the solar diurnal anisotropy, the data are divided into five periods: 2000 2001, 2002-2003, 2004-2005, 2006-2007 and 2008-2009. 
Based on the "East-West" method, the amplitudes and phases of the mean solar diurnal anisotropy in the multi$\mathrm{TeV}$ region are summarized in Table 1 . We find the amplitudes at $4.0 \mathrm{TeV}$ approximately twice as large as what the CG effect predicts in each period, that is to say, there is a remarkable additional anisotropy component superposing on the constant CG anisotropy. This is consistent with the previous results reported by Amenomori et al. [5].

On the other hand, a spurious variation in the solar time frame can arise from the seasonal change of the sidereal diurnal variation owing to the galactic anisotropy. This sideband component, which is hard to eliminate, can be estimated as a systematic uncertainty of the solar daily anisotropy from the extended-sidereal diurnal variation. Table 2 presents the observed $A^{R}$ and $\Phi^{R}$ in the extendedsidereal time frame.

In order to reveal the correlation of the solar activity and the "additional" anisotropy clearly, we derive this residual component by subtracting the harmonic vector predicted by the CG effect (with an amplitude of $0.04 \%$ and a phase of 06:00 LT) from the observed solar time anisotropy. Since the "additional" anisotropy is observed in the solar time frame, we estimate its systematic error from the extendedsidereal time as we do above. Figure 1 and Figure 2 display the temporal variation of the amplitudes and phases of the "additional" anisotropy, respectively.

As shown in Figure 1, the amplitudes of the residual anisotropy observed by the Tibet experiment are consistent with being stable between the maximum and minimum of the 23rd solar cycle. To evaluate the stability of the results, each data set is fitted with a flat line. Table 3 shows the $\chi^{2}$-fitted results of the amplitudes, taking into account the systematic errors. Therefore, the average amplitudes of the additional anisotropy can be calculated as $0.041 \% \pm 0.003 \%$ at $4.0 \mathrm{TeV}, 0.013 \% \pm 0.004 \%$ at 6.2 $\mathrm{TeV}$ and $0.013 \% \pm 0.004 \%$ at $12.0 \mathrm{TeV}$, respectively. It is noted that the amplitude at $4.0 \mathrm{TeV}$ has the same order of magnitude as the expected CG anisotropy and it decreases at higher energies. On the contrary, there is a significant difference from the evolution of the amplitudes observed by the Matsushiro experiment at $0.6 \mathrm{TeV}$, which varies between a maximum of $0.050 \% \pm 0.010 \%$ and a minimum of $0.002 \% \pm 0.009 \%$ and behaves similarly to the temporal variation of the monthly mean sunspot number when taking into account a 26 months time lag effect. This time lag corresponds to the transit time of the solar wind propagating through $\sim 180 \mathrm{AU}$, which implies the $0.6 \mathrm{TeV}$ galactic cosmic rays are subject to the solar modulation over the entire region within, and possible beyond, the heliospheric termination shock.

The amplitude and phase are two essential features for describing an anisotropic harmonic vector. It is also neccessary to examine the evolution of the phases of the "additional" anisotropy. Figure 2 shows the temporal variation of the phases of this extra effect observed by the Tibet and the Matsushiro experiment, respectively. One can see that all the phases are almost unchanged from 2000 to 2009. Note that the amplitudes at $6.2 \mathrm{TeV}$ and $12 \mathrm{TeV}$ are below $0.015 \%$ in 2002-2003 and 2008-2009, thus these insignificant anisotropies could result in large

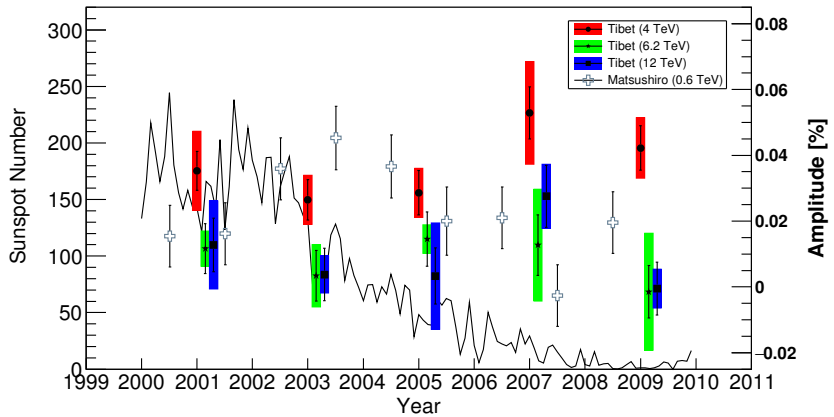

Figure 1: The amplitudes of the "additional" anisotropy observed by the Tibet experiment at $4.0 \mathrm{TeV}$ (solid circles), $6.2 \mathrm{TeV}$ (solid pentagrams) and $12 \mathrm{TeV}$ (solid squares), along with Matsushiro's results at $0.6 \mathrm{TeV}$ (open crosses). Error boxes for the Tibet experiment indicate systematic errors obtained from analyzing the same data in the extended-sidereal time frame, while the error bars are statistical. The errors of Matsushiro's results are statistical only. The gray line indicates the monthly mean sunspot number.

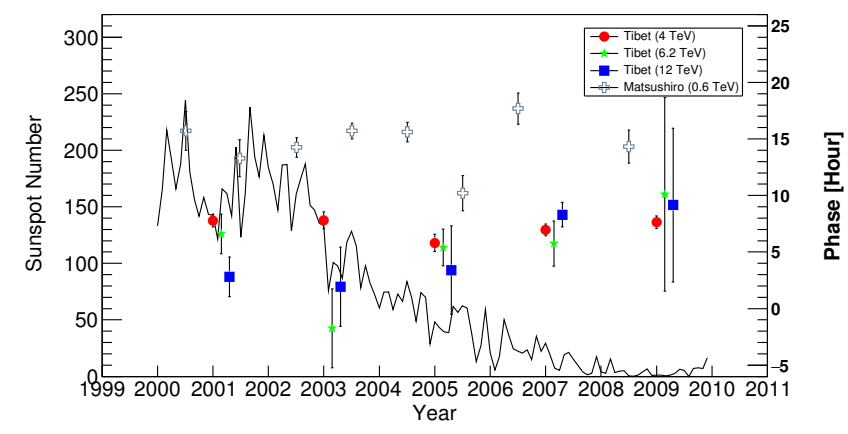

Figure 2: The phases of the "additional" anisotropy observed by the Tibet experiment at $4.0 \mathrm{TeV}$ (red circles), $6.2 \mathrm{TeV}$ (green pentagrams) and $12 \mathrm{TeV}$ (blue squares), in comparison with the Matsushiro experiment at $0.6 \mathrm{TeV}$ (gray crosses). The errors are all statistical only. The yearly mean phase observed by Matsushiro in 2007 is omitted in order to exclude large fluctuation due to the insignificant anisotropy. The gray line indicates the monthly mean sunspot number.

fluctuations on the phases. The results of fitting the phases with a flat line are summarized in Table 3 , together with the amplitudes. It can be seen that the phases observed by the Tibet experiment are fairly constant around 07:17 \pm 00:16 LT at 4.0 TeV, 05:22 \pm 00:58 LT at $6.2 \mathrm{TeV}$ and 06:20 \pm 00:52 LT at $12.0 \mathrm{TeV}$, respectively. As for the Matsushiro experiment, the phase becomes about 15:10 \pm 00:25 LT at $0.6 \mathrm{TeV}$, which is nearly twice as large as that measured by Tibet.

\section{Conclusions}

We analyze the temporal variations of the solar diurnal anisotropy in the multi- $\mathrm{TeV}$ cosmic-ray intensity observed by the Tibet experiment from 2000 to 2009 . There is a significant extra anisotropy superimposed on the Compton-Getting anisotropy at $4.0 \mathrm{TeV}$ while the extra anisotropy decreases at higher energy regions. In contrast to the additional anisotropy reported by the Matsushiro experiment at $0.6 \mathrm{TeV}$, the additional anisotropy measured by Tibet at multi-TeV energies is consistent with being stable, with the amplitude of $0.04 \%$ and the phase of $7 \mathrm{hr}$ 
Table 1: Amplitudes and Phases of the Multi-TeV Mean Solar Diurnal Variation Observed by Tibet in 2000-2009

\begin{tabular}{|c|c|c|c|c|c|c|}
\hline \multirow{2}{*}{ Period } & \multicolumn{2}{|c|}{$4.0 \mathrm{TeV}$} & \multicolumn{2}{|c|}{$6.2 \mathrm{TeV}$} & \multicolumn{2}{|c|}{$12.0 \mathrm{TeV}$} \\
\hline & $A^{R}\left(\times 10^{-3} \%\right)$ & $\Phi^{R}(h)$ & $A^{R}\left(\times 10^{-3} \%\right)$ & $\Phi^{R}(h)$ & $A^{R}\left(\times 10^{-3} \%\right)$ & $\Phi^{R}(h)$ \\
\hline 2000-2001 & $78.1 \pm 5.9$ & $7.15 \pm 0.29$ & $55.6 \pm 7.6$ & $6.53 \pm 0.52$ & $51.2 \pm 8.1$ & $5.40 \pm 0.61$ \\
\hline 2002-2003 & $69.4 \pm 6.2$ & $7.08 \pm 0.34$ & $35.0 \pm 7.7$ & $5.74 \pm 0.84$ & $43.0 \pm 8.0$ & $5.77 \pm 0.71$ \\
\hline 2004-2005 & $72.3 \pm 6.7$ & $6.18 \pm 0.36$ & $57.9 \pm 8.2$ & $6.14 \pm 0.55$ & $45.1 \pm 8.5$ & $5.99 \pm 0.72$ \\
\hline 2006-2007 & $96.7 \pm 7.9$ & $6.78 \pm 0.31$ & $56.4 \pm 9.3$ & $6.26 \pm 0.63$ & $69.5 \pm 9.4$ & $7.32 \pm 0.51$ \\
\hline 2008-2009 & $85.2 \pm 6.7$ & $7.12 \pm 0.30$ & $41.1 \pm 7.9$ & $6.77 \pm 0.74$ & $42.5 \pm 8.0$ & $6.76 \pm 0.72$ \\
\hline
\end{tabular}

Table 2: Amplitudes and Phases of the Multi-TeV Mean Extended-sidereal Diurnal Variation Observed by Tibet in 2000-2009

\begin{tabular}{|c|c|c|c|c|c|c|}
\hline \multirow{2}{*}{ Period } & \multicolumn{2}{|c|}{$4.0 \mathrm{TeV}$} & \multicolumn{2}{|c|}{$6.2 \mathrm{TeV}$} & \multicolumn{2}{|c|}{$12.0 \mathrm{TeV}$} \\
\hline & $A^{R}\left(\times 10^{-3} \%\right)$ & $\Phi^{R}(h)$ & $A^{R}\left(\times 10^{-3} \%\right)$ & $\Phi^{R}(h)$ & $A^{R}\left(\times 10^{-3} \%\right)$ & $\Phi^{R}(h)$ \\
\hline 2000-2001 & $12.0 \pm 5.9$ & $4.90 \pm 1.89$ & $5.3 \pm 7.6$ & $7.48 \pm 5.45$ & $13.4 \pm 8.1$ & $9.69 \pm 2.31$ \\
\hline 2002-2003 & $7.4 \pm 6.2$ & $20.79 \pm 3.16$ & $9.5 \pm 7.7$ & $3.58 \pm 3.09$ & $5.6 \pm 8.0$ & $22.77 \pm 5.46$ \\
\hline 2004-2005 & $7.5 \pm 6.7$ & $22.06 \pm 3.43$ & $4.3 \pm 8.2$ & $18.86 \pm 7.29$ & $16.1 \pm 8.5$ & $12.24 \pm 2.02$ \\
\hline 2006-2007 & $15.6 \pm 7.9$ & $19.44 \pm 1.92$ & $17.0 \pm 9.3$ & $4.58 \pm 2.07$ & $9.8 \pm 9.4$ & $20.87 \pm 3.66$ \\
\hline 2008-2009 & $9.2 \pm 6.7$ & $17.42 \pm 2.79$ & $17.8 \pm 7.9$ & $3.21 \pm 1.71$ & $5.9 \pm 8.0$ & $19.66 \pm 5.19$ \\
\hline
\end{tabular}

Table 3: Fitting Results of the Amplitudes and Phases of the Multi-TeV Additional Anisotropy with a Flat Line

\begin{tabular}{ccccc}
\hline \hline $\begin{array}{c}\text { Energy } \\
(\mathrm{TeV})\end{array}$ & $\begin{array}{c}A_{\text {fit }}\left(\times 10^{-3} \%\right) \\
(\text { stat. }+ \text { sys. })\end{array}$ & $\chi^{2} /$ d.o.f. & $\begin{array}{c}\Phi_{f i t}(h) \\
(\text { stat. })\end{array}$ & $\chi^{2} /$ d.o.f. \\
\hline 4.0 & $40.7 \pm 2.9$ & $9.0 / 4$ & $7.28 \pm 0.27$ & $5.8 / 4$ \\
6.2 & $12.8 \pm 3.6$ & $2.9 / 4$ & $5.37 \pm 0.97$ & $5.0 / 4$ \\
12 & $13.4 \pm 3.7$ & $6.3 / 4$ & $6.34 \pm 0.87$ & $9.6 / 4$ \\
\hline
\end{tabular}

local solar time. Since the extra solar anisotropy observed by the Tibet experiment does not show a clear correlation with the solar activity, it suggests that these two additional anisotropy component mentioned above could result from different mechanisms.

\section{Acknowledgements}

The collaborative experiment of the Tibet Air Shower Arrays has been performed under the auspices of the Ministry of Science and Technology of China and the Ministry of Foreign Affairs of Japan. This work is supported by the National Key R\&D Program of China (No. 2016YFE0125500), by the Grants from the National Natural Science Foundation of China (Nos. 11533007, 11673041, 11603047 and 11275212), and by the Key Laboratory of Particle Astrophysics, Institute of High Energy Physics, CAS. This work is supported by the joint research program of the Institute for Cosmic Ray Research (ICRR), the University of Tokyo.

\section{References}

[1] A.H. Compton \& I.A. Getting, The Physical Review 47, 817 (1935)

[2] L.J. Gleeson \& W.I. Axford, Astrophysics and Space Science 2, 431 (1968)

[3] D.J. Cutler \& D.E. Groom, Nature 322, 434 (1986)

[4] M. Aglietta et al., The Astrophysical Journal 470, 501 (1996)

[5] M. Amenomori et al., Physical Review Letters 93, 061101 (2004)

[6] M. Amenomori et al., Science 314, 439 (2006)

[7] M. Aglietta et al., The Astrophysical Journal 692, L130 (2009)

[8] K. Munakata et al., The Astrophysical Journal 712, 1100 (2010)

[9] R. Abbasi et al., The Astrophysical Journal 746, 33 (2012)

[10] M. Amenomori et al., Physical Review Letters 69, 2468 (1992)

[11] M. Amenomori et al., The Astrophysical Journal 678, 1165 (2008)

[12] K. Nagashima et al., Il Nuovo Cimento 12C, 695 (1989) 